1 Secretaria de Estado de Saúde de Mato Grosso do Sul - Campo Grande (MS), Brasil.

karine.costa@saude.ms.gov.br

2 Universidade Federal de Mato Grosso do Sul (UFMS), Centro de Ciências Biológicas e da Saúde, Programa de Pós-Graduação em Saúde da Família - Campo Grande (MS), Brasil.

luizacazola@gmail.com

3 Universidade Federal de Mato Grosso do Sul (UFMS), Centro de Ciências Biológicas e da Saúde - Campo Grande (MS), Brasil.

edson.tamaki@ufms.br

\section{Assessment of Chronic Illness Care (ACIC): avaliação da aplicabilidade e resultados}

\author{
Assessment of Chronic Illness Care (ACIC): evaluation of \\ applicability and results
}

Karine Cavalcante da Costa', Luiza Helena de Oliveira Cazola ${ }^{\mathbf{2}}$, Edson Mamoru Tamakiª

RESUMO Estudo descritivo de abordagem quanti-qualitativa, cujo objetivo foi avaliar a aplicação do Assessment of Chronic Illness Care (ACIC) e seus resultados junto aos profissionais de equipes da Estratégia Saúde da Família, em Campo Grande (MS). A amostra constituiu-se de 30 profissionais em 5 equipes, e a coleta de dados foi realizada em maio e junho de 2014, por meio do instrumento ACIC. A média das equipes resultou em capacidade razoável para atenção às condições crônicas, sendo o sistema de informação clínica a principal fragilidade e o desenho do sistema de prestação de serviços de saúde, o maior potencial.

PALAVRAS-CHAVE Atenção Primária à Saúde; Avaliação de serviços de saúde; Doenças crônicas; Estratégia Saúde da Família.

ABSTRACT Descriptive study of quantitative and qualitative approach, sought to evaluate the application of the Assessment of Chronic Illness Care (ACIC) and its results along with professionals of teams of the Family Health Strategy, in Campo Grande (MS). The sample consisted of 30 professionals in 5 teams, and data collection was carried out in May and June of 2014, through the application of ACIC instrument. The average of the teams resulted in reasonable ability to the care for chronic conditions, and the clinical information system as the main weakness and the drawing of the healthcare service delivery system, the greatest potential.

KEYWORDS Primary Health Care; Health services evaluation; Chronic diseases; Family Health Strategy. 


\section{Introdução}

As condições crônicas são consideradas as principais causas de morbimortalidade no mundo, sendo responsáveis por $58 \%$ das causas de óbito, devido ao envelhecimento da população e às mudanças nos hábitos de vida: sedentarismo, alimentação inadequada, tabagismo e estratégias mercadológicas (ALWAN ET AL., 2010). No Brasil, se observa que as doenças cardiovasculares, neoplasias e doenças respiratórias representam $72 \%$ das causas de óbitos. O País passa por um envelhecimento da população e pelo crescimento do número de pessoas em sobrepeso e obesidade (DUARTE; BARRETO, 2012).

Estas condições exigem respostas e ações contínuas, proativas e integradas, desde o sistema de atenção à saúde e dos profissionais até os usuários, para a estabilização das condições de saúde por meio de um controle efetivo, eficiente e com qualidade (MENDES, 2012; MACINKO; DOURADO; GUANAIS, 2011). O sistema de saúde, no Brasil, para atender a estas transformações, vive em um descompasso entre o aumento das condições crônicas e o processo de trabalho e da gestão, que ainda privilegiam as condições agudas ou a agudização das condições crônicas (BRASIL, 2011).

Também se observa que os sistemas de saúde, além de terem um papel essencial na prevenção das condições crônicas, bem como nas estratégias de controle, devem se articular com outros setores e atores. Portanto, o modelo de atenção adotado é determinante para o sucesso da melhoria das condições de vida de um indivíduo ou comunidade, em especial, o modelo de atenção às condições crônicas (MACINKO; DOURADO; GUANAIS, 2011; MOYSÉS; SILVEIRA FILHO; MOYSÉS, 2012).

A estratégia existente no Brasil para superar a fragmentação da atenção e da gestão nas regiões de saúde e aperfeiçoar o funcionamento político institucional do Sistema Único de Saúde (SUS), com vistas a assegurar ao usuário o conjunto de ações e serviços efetivos e eficientes dos quais necessitam, é a Rede de Atenção à Saúde (RAS). O pilar de uma RAS é a Atenção Primária à Saúde (APS), que assume o papel central no desenho e no funcionamento desses sistemas. Portanto, o sucesso da RAS depende da efetividade da APS, por meio dos seus atributos: resolutividade, responsabilização e coordenação (BRASIL, 2010).

Para organizar os pontos de atenção às condições crônicas no Brasil, foi publicada pelo Ministério da Saúde/Gabinete do Ministro (MS/GM) a Portaria $n^{\circ} 483 / 2014$, que estabelece a RAS das pessoas com doenças crônicas no âmbito do SUS, com seus componentes, diretrizes e objetivos (BRASIL, 2014).

A fim de estabelecer intervenções resolutivas para a mudança do perfil epidemiológico, o MacColl Institute for Health Inovation, nos Estados Unidos, criou o Modelo de Atenção Crônica, mais conhecido como Modelo de Condições Crônicas (MCC), que traz importantes conceitos para a melhoria do processo de trabalho a essas condições (WAGNER, 1998; DAHLGREN; WHITEHEAD, 1991). Mendes (2012) fez uma adaptação desse modelo, unindo-o ao Modelo da Pirâmide de Risco e ao Modelo da Kaiser Permanente, criando o Modelo de Atenção às Condições Crônicas (MACC).

Para avaliação da implantação do MCC foi construído o instrumento Assessment of Chronic Illness Care (ACIC) (Avaliação da Capacidade Institucional para a Atenção às Condições Crônicas), que tem a finalidade de avaliar a atenção às condições crônicas por meio da percepção dos profissionais, e deve ser utilizado pelas equipes de saúde para identificar áreas com maior fragilidade, a fim de qualificar a atenção em doenças crônicas antes da programação de ações para a qualificação e avaliar o nível das intervenções adotadas (BONOMI ET AL, 2002).

Esse instrumento foi traduzido e adaptado transculturalmente para diversas línguas, inclusive para o português (BOWEN ET AL, 2010; CRAMM ET AL, 2011; GOMTUBUTRA ET AL, 2012). A Secretaria Municipal de Saúde de Curitiba 
(PR), em parceria com o Laboratório de Inovações no Cuidado às Condições Crônicas, a Organização Pan-Americana da Saúde (Opas) e o Conselho Nacional de Secretários de Saúde (Conass), utilizou o ACIC para a implantação do MACC. O instrumento foi validado e aplicado em dois momentos: no primeiro, para definição de uma linha de base, para posterior implantação do MACC; e no segundo, a sua reaplicação para comparação com a linha de base (MOYSÉs; SILVEIRA FILHO; MOYSÉS, 2012).

Com este estudo, pretende-se avaliar a primeira aplicação do instrumento ACIC aos profissionais de equipes da Estratégia Saúde da Família (ESF) de Campo Grande (MS), para estabelecimento da linha de base, antecedendo a implantação da Rede de Atenção à Saúde das Pessoas com Doenças Crônicas, bem como os resultados obtidos, que demonstrarão a capacidade institucional para atenção às condições crônicas dessas equipes.

\section{Metodologia}

Trata-se de pesquisa descritiva com abordagem quanti-qualitativa baseada em dados primários, obtidos por meio da aplicação do ACIC aos profissionais que compõem as equipes da ESF urbanas, de Campo Grande (MS).

Foram considerados como critérios para inclusão: a existência de Unidades Básicas de Saúde da Família (UBSF), compostas pelas equipes mínimas, conforme Brasil (2012), com médico, enfermeiro, auxiliar ou técnico de enfermagem, agentes comunitários de saúde e cirurgião-dentista, além de auxiliar e/ou técnico de saúde bucal; terem sido implantadas até dezembro de 2012, por ser necessário um tempo mínimo de conhecimento da população adscrita; e a presença de, no mínimo, três profissionais, sendo obrigatória a participação do(a) enfermeiro(a) e do(a) médico(a).
Para avaliação da aplicabilidade do instrumento foram consideradas as seguintes variáveis: tempo de aplicação, número de participantes e métodos de aplicação, conceitos baseados na literatura (MOYSÉS; SILVEIRA FILHO; MOYSÉs, 2012). Como critérios de exclusão, equipes cujos profissionais não aceitassem participar da pesquisa, equipes incompletas e aquelas nas quais os profissionais médicos e enfermeiros estivessem afastados, por qualquer motivo (férias e licença).

Para a escolha das equipes, dentre um universo de 84 implantadas, foi utilizada a amostragem probabilística por conglomerados, e procedeu-se o sorteio de uma UBSF em cada um dos quatro distritos sanitários existentes em Campo Grande (MS).

O ACIC é composto por seis dimensões associadas à efetivação do MACC, e uma sétima, que avalia a integração das dimensões. As percepções obtidas são analisadas por meio de uma escala de pontuação, de 0 a 11, em que (0) representa a menor pontuação, ou seja, um local com recursos e estruturas muito limitados; e (11), a maior pontuação, um local com recursos e estrutura ótima para a atenção às condições crônicas. Para cada dimensão, é obtida uma pontuação média e, ao final, é feita sua soma e dividida pelo número de dimensões (MOYSÉS; SILVEIRA FILHO; MOYSÉS, 2012).

As sete dimensões do instrumento ACIC são: organização da atenção à saúde, articulação com a comunidade, autocuidado apoiado, suporte à decisão, desenho do sistema de prestação de serviços, sistema de informação clínica e integração dos componentes do MACC. Cada uma constitui-se de quatro ou seis componentes (quadro 1).

Sua aplicação ocorreu nos meses de maio a junho de 2014, durante a reunião da equipe, que contou com a presença da pesquisadora e foi agendado previamente com o gerente da unidade de saúde, para que não houvesse comprometimento no processo de trabalho. Participaram 30 profissionais de distintas categorias, lotados em 5 equipes de ESF (tabela $1)$. 
Quadro 1. Componentes das sete dimensões do ACIC

\begin{tabular}{|c|c|c|c|c|c|c|}
\hline \multicolumn{7}{|c|}{ Componentes das Dimensões } \\
\hline $\begin{array}{l}\text { 1: Organização } \\
\text { da Atenção à } \\
\text { Saúde }\end{array}$ & $\begin{array}{c}\text { 2: Articulação } \\
\text { com a } \\
\text { Comunidade }\end{array}$ & $\begin{array}{l}\text { 3: Auto- } \\
\text { cuidado } \\
\text { Apoiado }\end{array}$ & $\begin{array}{l}\text { 4: Suporte } \\
\text { à Decisão }\end{array}$ & $\begin{array}{l}\text { 5: Desenho } \\
\text { do Sistema de } \\
\text { Prestação de } \\
\text { Serviços }\end{array}$ & $\begin{array}{l}\text { 6: Sistema de } \\
\text { Informação } \\
\text { Clínica }\end{array}$ & $\begin{array}{c}\text { 7: Integração dos } \\
\text { Componentes do } \\
\text { MACC }\end{array}$ \\
\hline $\begin{array}{l}\text { Existência e } \\
\text { incorporação } \\
\text { do interesse } \\
\text { da liderança } \\
\text { organizacional } \\
\text { em relação } \\
\text { às mudanças } \\
\text { na atenção } \\
\text { às condições } \\
\text { crônicas. }\end{array}$ & $\begin{array}{l}\text { Realização de } \\
\text { articulação } \\
\text { das institui- } \\
\text { ções de saúde } \\
\text { e das pessoas } \\
\text { usuárias com } \\
\text { os recursos } \\
\text { comunitários. }\end{array}$ & $\begin{array}{l}\text { Realização } \\
\text { de avaliação } \\
\text { e documen- } \\
\text { tação das } \\
\text { atividades } \\
\text { e necessi- } \\
\text { dades de } \\
\text { autocuidado } \\
\text { apoiado. }\end{array}$ & $\begin{array}{l}\text { Disponibi- } \\
\text { lidade de } \\
\text { diretrizes } \\
\text { clínicas } \\
\text { baseadas } \\
\text { em evi- } \\
\text { dências. }\end{array}$ & $\begin{array}{l}\text { Trabalho em } \\
\text { equipe, que se } \\
\text { reúne com atri- } \\
\text { buições bem } \\
\text { definidas. }\end{array}$ & $\begin{array}{l}\text { Disponibilida- } \\
\text { de de pron- } \\
\text { tuário clínico } \\
\text { eletrônico. }\end{array}$ & $\begin{array}{l}\text { Realização de } \\
\text { informação às } \\
\text { pessoas usuárias } \\
\text { em relação às dire- } \\
\text { trizes clínicas. }\end{array}$ \\
\hline $\begin{array}{l}\text { Existência e } \\
\text { monitoramen- } \\
\text { to das metas } \\
\text { organizacionais } \\
\text { para a atenção } \\
\text { às condições } \\
\text { crônicas. }\end{array}$ & $\begin{array}{l}\text { Parcerias com } \\
\text { organizações } \\
\text { comunitárias } \\
\text { existentes, } \\
\text { estruturadas e } \\
\text { pró-ativas. }\end{array}$ & $\begin{array}{l}\text { Oferecimen- } \\
\text { to, pelos } \\
\text { educadores } \\
\text { em saúde, } \\
\text { de suporte } \\
\text { para o au- } \\
\text { tocuidado } \\
\text { apoiado. }\end{array}$ & $\begin{array}{l}\text { Envolvi- } \\
\text { mento de } \\
\text { especia- } \\
\text { listas no } \\
\text { apoio à } \\
\text { Atenção } \\
\text { Primária à } \\
\text { Saúde. }\end{array}$ & $\begin{array}{l}\text { Liderança } \\
\text { das equipes } \\
\text { de saúde } \\
\text { reconhecida, } \\
\text { assumida e } \\
\text { garantida pela } \\
\text { instituição de } \\
\text { saúde. }\end{array}$ & $\begin{array}{l}\text { Disponibilida- } \\
\text { de de registro } \\
\text { das pessoas } \\
\text { usuárias (lista } \\
\text { de pessoas } \\
\text { com condi- } \\
\text { ções crônicas } \\
\text { específicas } \\
\text { por estrato de } \\
\text { risco). }\end{array}$ & $\begin{array}{l}\text { Sistema de infor- } \\
\text { mação/registro } \\
\text { clínico com a } \\
\text { inclusão de resul- } \\
\text { tados de avaliação } \\
\text { do estado das } \\
\text { pessoas usuárias. }\end{array}$ \\
\hline $\begin{array}{l}\text { Existência e } \\
\text { incorporação } \\
\text { das estratégias } \\
\text { para a melhoria } \\
\text { da atenção } \\
\text { às condições } \\
\text { crônicas. }\end{array}$ & $\begin{array}{l}\text { Existência do } \\
\text { Conselho Lo- } \\
\text { cal de Saúde } \\
\text { e acompa- } \\
\text { nhamento } \\
\text { do mesmo à } \\
\text { atenção às } \\
\text { condições } \\
\text { crônicas. }\end{array}$ & $\begin{array}{l}\text { Acolhimento } \\
\text { das preo- } \\
\text { cupações } \\
\text { das pessoas } \\
\text { usuárias } \\
\text { e seus } \\
\text { familiares } \\
\text { como parte } \\
\text { integrante } \\
\text { da atenção } \\
\text { às condições } \\
\text { crônicas. }\end{array}$ & $\begin{array}{l}\text { Realização } \\
\text { de educa- } \\
\text { ção per- } \\
\text { manente } \\
\text { dos pro- } \\
\text { fissionais } \\
\text { de saúde } \\
\text { para } \\
\text { atenção às } \\
\text { condições } \\
\text { crônicas. }\end{array}$ & $\begin{array}{l}\text { Sistema de } \\
\text { agendamento } \\
\text { organizado. }\end{array}$ & $\begin{array}{l}\text { Disponibi- } \\
\text { lidade de } \\
\text { alerta para os } \\
\text { profissionais, } \\
\text { de forma a } \\
\text { permitir a } \\
\text { identificação } \\
\text { de subpopu- } \\
\text { lações por } \\
\text { estratificação } \\
\text { de risco. }\end{array}$ & $\begin{array}{l}\text { Programas co- } \\
\text { munitários que } \\
\text { fornecem feedback } \\
\text { à instituição de } \\
\text { saúde sobre as } \\
\text { pessoas usuárias. }\end{array}$ \\
\hline $\begin{array}{l}\text { Utilização da } \\
\text { regulação e de } \\
\text { incentivos para } \\
\text { apoiar a aten- } \\
\text { ção às condi- } \\
\text { ções crônicas. }\end{array}$ & $\begin{array}{l}\text { Incorporação } \\
\text { do agente } \\
\text { comunitário } \\
\text { de saúde à } \\
\text { atenção às } \\
\text { condições } \\
\text { crônicas. }\end{array}$ & $\begin{array}{l}\text { Disponibi- } \\
\text { lização de } \\
\text { intervenções } \\
\text { efetivas de } \\
\text { mudança } \\
\text { de compor- } \\
\text { tamento } \\
\text { de pessoas } \\
\text { usuárias e } \\
\text { de suporte } \\
\text { de pares. }\end{array}$ & $\begin{array}{l}\text { Informa- } \\
\text { ção às } \\
\text { pessoas } \\
\text { usuárias } \\
\text { sobre as } \\
\text { diretrizes } \\
\text { clínicas } \\
\text { realizadas } \\
\text { por meio } \\
\text { de méto- } \\
\text { dos ade- } \\
\text { quados de } \\
\text { educação } \\
\text { em saúde. }\end{array}$ & $\begin{array}{l}\text { Monitoramen- } \\
\text { to da condição } \\
\text { crônica oferta- } \\
\text { do e organiza- } \\
\text { do pela equipe } \\
\text { de saúde e } \\
\text { adaptado às } \\
\text { necessidades } \\
\text { das pessoas } \\
\text { usuárias. }\end{array}$ & $\begin{array}{l}\text { Feedbacks } \\
\text { fornecidos } \\
\text { oportuna- } \\
\text { mente para } \\
\text { a equipe de } \\
\text { saúde. }\end{array}$ & $\begin{array}{l}\text { Utilização de } \\
\text { dados dos siste- } \\
\text { mas de informação } \\
\text { para planejamento } \\
\text { de programação } \\
\text { local das condi- } \\
\text { ções crônicas. }\end{array}$ \\
\hline $\begin{array}{l}\text { Existência } \\
\text { de liderança } \\
\text { superior da } \\
\text { organização, } \\
\text { que encoraja } \\
\text { a melhoria } \\
\text { da atenção } \\
\text { às condições } \\
\text { crônicas. }\end{array}$ & - & - & - & $\begin{array}{l}\text { Atenção pro- } \\
\text { gramada para } \\
\text { as condições } \\
\text { crônicas, uti- } \\
\text { lizada para a } \\
\text { maioria das } \\
\text { pessoas usuá- } \\
\text { rias. }\end{array}$ & $\begin{array}{l}\text { Disponibi- } \\
\text { lização de } \\
\text { informação } \\
\text { sobre subgru- } \\
\text { pos relevantes } \\
\text { de pessoas } \\
\text { usuárias, para } \\
\text { ajuda os pro- } \\
\text { fissionais no } \\
\text { planejamento } \\
\text { do cuidado. }\end{array}$ & $\begin{array}{l}\text { Monitoramento } \\
\text { das metas dos } \\
\text { planos de cuidado } \\
\text { realizado por um } \\
\text { profissional desig- } \\
\text { nado, que utiliza } \\
\text { prontuário clínico e } \\
\text { alertas. }\end{array}$ \\
\hline
\end{tabular}

Fonte: Elaboração própria. 
Tabela 1. Quantitativo de profissionais participantes da aplicação do instrumento ACIC junto às equipes selecionadas da ESF de Campo Grande (MS), 2014

\begin{tabular}{|c|c|c|}
\hline Equipe & Total de Profissionais & Profissionais Participantes \\
\hline 01 & 09 & $\begin{array}{r}01 \text { médico } \\
01 \text { enfermeiro } \\
01 \text { dentista } \\
01 \text { auxiliar de enfermagem } \\
01 \text { auxiliar de saúde bucal } \\
04 \text { agentes comunitários de saúde }\end{array}$ \\
\hline 02 & 11 & $\begin{array}{r}01 \text { médico } \\
01 \text { enfermeiro } \\
01 \text { dentista } \\
01 \text { auxiliar de enfermagem } \\
01 \text { auxiliar de saúde bucal } \\
06 \text { agentes comunitários de saúde }\end{array}$ \\
\hline 03 & 04 & $\begin{array}{r}01 \text { médico } \\
01 \text { enfermeiro } \\
01 \text { auxiliar de enfermagem } \\
01 \text { agente comunitário de saúde }\end{array}$ \\
\hline 04 & 03 & $\begin{array}{r}01 \text { médico } \\
01 \text { enfermeiro } \\
01 \text { auxiliar de saúde bucal }\end{array}$ \\
\hline 05 & 03 & $\begin{array}{r}01 \text { médico } \\
01 \text { enfermeiro } \\
01 \text { auxiliar de enfermagem }\end{array}$ \\
\hline Total & 30 & $\begin{array}{r}05 \text { médicos } \\
05 \text { enfermeiros } \\
02 \text { dentistas } \\
04 \text { auxiliares de enfermagem } \\
03 \text { auxiliares de saúde bucal } \\
11 \text { agentes comunitários de saúde }\end{array}$ \\
\hline
\end{tabular}

Fonte: Elaboração própria.

Antecedendo a aplicação do instrumento, a pesquisadora apresentou o alinhamento conceitual em relação aos conceitos de doença e condição crônica, o impacto destas no perfil de morbimortalidade e a importância da APS na modificação deste perfil; também foram apresentados a relevância e objetivos da pesquisa, além de instruções em relação ao ACIC.

Após o aceite pelos participantes e a assinatura do termo de consentimento livre e esclarecido, iniciou-se a aplicação coletiva do instrumento ACIC. Para isso, foi necessário que a equipe pudesse estar em local reservado, a fim de discutir os questionamentos, bem como registrar os apontamentos. A pesquisadora distribuiu cópia do instrumento a cada participante, para que todos acompanhassem a leitura de cada componente, de maneira a facilitar a discussão. Dessa forma, as notas seriam dadas por consenso, prática recomendada por outros estudos (BONOMI ET AL., 2002).

Após a discussão do instrumento foram realizados cálculos para a obtenção da média final de todas as equipes, ou seja, a avaliação da capacidade institucional para atenção às condições crônicas, assim como a média final por equipe e por dimensões (quadro 2).

O estudo foi aprovado pelo Comitê de Ética em Pesquisa da Universidade Federal de Mato Grosso do Sul, sob o Protocolo de $\mathrm{n}^{\circ} 574.735$. 
Quadro 2. Dimensões e conceitos do ACIC

\begin{tabular}{|c|c|}
\hline Dimensões & Conceito \\
\hline Organização da Atenção à Saúde & $\begin{array}{l}\text { A atenção às condições crônicas pode ser mais efetiva se todo o sistema } \\
\text { (organização, instituição, unidade) no qual a atenção é prestada estiver } \\
\text { orientado e permitir maior ênfase no cuidado às condições crônicas. }\end{array}$ \\
\hline Articulação com a Comunidade & $\begin{array}{l}\text { A articulação entre o sistema de saúde (instituição/unidades básicas de } \\
\text { saúde) e os recursos comunitários tem um importante papel na gestão/ } \\
\text { manejo de condições crônicas. }\end{array}$ \\
\hline Autocuidado Apoiado & $\begin{array}{l}\text { O autocuidado apoiado efetivo pode ajudar as pessoas com condições } \\
\text { crônicas e suas famílias a lidar com os desafios de conviver e tratar a con- } \\
\text { dição crônica, além de reduzir as complicações e os sintomas da doença. }\end{array}$ \\
\hline Suporte à Decisão & $\begin{array}{l}\text { O manejo efetivo de condições crônicas assegura que os profissionais da } \\
\text { saúde tenham acesso a informações baseadas em evidências para apoiar } \\
\text { as decisões na atenção às pessoas usuárias. Isso inclui diretrizes e proto- } \\
\text { colos baseados em evidências, consultas a especialistas, educadores em } \\
\text { saúde, e envolvimento dos usuários, de forma a tornar as equipe de saúde } \\
\text { capazes de identificar estratégias efetivas de cuidado. }\end{array}$ \\
\hline $\begin{array}{l}\text { Desenho do Sistema de Prestação de } \\
\text { Serviços }\end{array}$ & $\begin{array}{l}\text { A evidência sugere que a gestão efetiva da atenção às condições crôni- } \\
\text { cas envolve mais do que a simples adição de intervenções a um sistema } \\
\text { focado no cuidado de condições agudás. São necessárias mudanças na } \\
\text { organização do sistema, realinhando a oferta do cuidado. }\end{array}$ \\
\hline Sistema de Informação Clínica & $\begin{array}{l}\text { Informação útil e oportuna, e personalizada, por cada usuário e pelas } \\
\text { populaçôes usuárias com condições crônicas, é um aspecto crítico de } \\
\text { modelos de atenção efetivos, especialmente daqueles que empregam } \\
\text { abordagens populacionais. }\end{array}$ \\
\hline $\begin{array}{l}\text { Integração dos Componentes do Modelo } \\
\text { de Atenção às Condições Crônicas }\end{array}$ & $\begin{array}{l}\text { Sistemas de saúde efetivos integram e combinam todos os elementos } \\
\text { do modelo, por exemplo, associando as metas de autocuidado com os } \\
\text { registros nos sistemas de informação, ou associando políticas locais com } \\
\text { atividades dos planos terapêuticos dos pacientes (locais para desenvolvi- } \\
\text { mento de atividades físicas, estruturação de hortas comunitárias etc.). }\end{array}$ \\
\hline
\end{tabular}

Interpretação dos resultados

Pontuações entre " $\mathrm{O}^{\prime \prime} \mathrm{e}$ " 2 ": capacidade limitada para a atenção às condições crônicas;

Pontuações entre "3" e "5": capacidade básica para a atenção às condiç̃̃es crônicas;

Pontuações entre " 6 " e " 8 ": razoável capacidade para a atenção às condicõos crônicas

Pontuações entre "9" e "11": capacidade ótima para a atenção às condições crônicas.

Fonte: MOYSÉS; SILVEIRA FILHO; MOYSÉS, 2012.

\section{Resultados e discussão}

Os resultados demonstraram que, ao se considerar a média final por equipe, variações foram encontradas, apresentando a equipe 4 a menor média $(3,15)$, interpretada como capacidade básica para atenção às condições crônicas e diferindo das demais, que apresentaram razoável capacidade (tabela 2).

Tabela 2. Resultados da aplicação do ACIC, médias por equipe, por dimensão e interpretação, em Campo Grande (MS), 2014

\begin{tabular}{|c|c|c|c|c|c|c|c|c|c|}
\hline \multirow[b]{2}{*}{ Equipes } & \multicolumn{7}{|c|}{ Dimensões } & \multirow[b]{2}{*}{$\begin{array}{l}\text { Média } \\
\text { Final } \\
\text { por } \\
\text { Equi- } \\
\text { pe }\end{array}$} & \multirow[b]{2}{*}{$\begin{array}{l}\text { Interpreta- } \\
\text { ção }\end{array}$} \\
\hline & $\begin{array}{l}\text { 1. Organi- } \\
\text { zação da } \\
\text { Atenção à } \\
\text { Saúde }\end{array}$ & $\begin{array}{l}\text { 2. Arti- } \\
\text { culação } \\
\text { com a } \\
\text { Comuni- } \\
\text { dade }\end{array}$ & $\begin{array}{l}\text { 3. Auto- } \\
\text { cuidado } \\
\text { Apoia- } \\
\text { do }\end{array}$ & $\begin{array}{l}\text { 4. Su- } \\
\text { porte à } \\
\text { Decisão }\end{array}$ & $\begin{array}{l}\text { 5. Dese- } \\
\text { nho do } \\
\text { Sistema } \\
\text { de } \\
\text { Presta- } \\
\text { ção de } \\
\text { Servi- } \\
\text { ços }\end{array}$ & $\begin{array}{l}\text { 6. Sis- } \\
\text { tema de } \\
\text { Infor- } \\
\text { mação } \\
\text { Clínica }\end{array}$ & $\begin{array}{l}7 . \\
\text { Inte- } \\
\text { gração } \\
\text { dos } \\
\text { Com- } \\
\text { ponen- } \\
\text { tes do } \\
\text { MACC }\end{array}$ & & \\
\hline Equipe 1 & 8,83 & 9 & 7,5 & 9,25 & 9,83 & 5 & 6 & 7,92 & $\begin{array}{r}\text { Razoável } \\
\text { capacida- } \\
\text { de para } \\
\text { atenção às } \\
\text { condições } \\
\text { crônicas }\end{array}$ \\
\hline
\end{tabular}




\begin{tabular}{|c|c|c|c|c|c|c|c|c|c|}
\hline Equipe 2 & 9,33 & 10,25 & 10 & 8,75 & 10,17 & 4,17 & 10,17 & 8,98 & $\begin{array}{r}\text { Razoável } \\
\text { capacida- } \\
\text { de para } \\
\text { atenção às } \\
\text { condições } \\
\text { crônicas }\end{array}$ \\
\hline Equipe 3 & 9,83 & 8 & 9,75 & 7,25 & 9,33 & 4 & 8,67 & 8,12 & $\begin{array}{r}\text { Razoável } \\
\text { capacida- } \\
\text { de para } \\
\text { atenção às } \\
\text { condições } \\
\text { crônicas }\end{array}$ \\
\hline Equipe 4 & 3,50 & 2,5 & 2,75 & 3,5 & 5,67 & 1,83 & 2,33 & 3,15 & $\begin{array}{r}\text { Capacidade } \\
\text { básica para } \\
\text { atenção às } \\
\text { condições } \\
\text { crônicas }\end{array}$ \\
\hline Equipe 5 & 7,83 & 7 & 8,25 & 5,67 & 7,83 & 5,33 & 0,67 & 6,02 & $\begin{array}{r}\text { Razoável } \\
\text { capacida- } \\
\text { de para } \\
\text { atenção às } \\
\text { condiç̃̃es } \\
\text { crônicas }\end{array}$ \\
\hline $\begin{array}{l}\text { Média } \\
\text { Final } \\
\text { das } \\
\text { Dimen- } \\
\text { sões }\end{array}$ & 7,87 & 7,35 & 7,65 & 6,80 & 8,57 & 4,07 & 5,57 & 6,84 & $\begin{array}{l}\text { Razoável } \\
\text { capacida- } \\
\text { de para } \\
\text { atenção às } \\
\text { condiç̃̃es } \\
\text { crônicas }\end{array}$ \\
\hline
\end{tabular}

Em relação à primeira equipe, observou-se, durante a aplicação do instrumento, uma participação ativa dos seus membros, promovida principalmente pela enfermeira, que possibilitou a integração do grupo (tabela 1).

A maior nota foi relacionada à quinta dimensão, desenho do sistema de prestação de serviços (tabela 2), pois os componentes trabalho em equipe, liderança das equipes de saúde e atenção programada para as condições crônicas obtiveram a totalidade dos pontos (quadro 1). O comportamento ativo desta equipe pode ter proporcionado um bom resultado nesta dimensão, pois as ações referentes à mesma estão no âmbito de atuação da equipe.

Esta dimensão traz a necessidade de que a gestão efetiva da atenção às condições crônicas envolva mudanças na organização do sistema no sentido de realinhar a oferta do cuidado; que haja intervenções no trabalho em equipe, na liderança das mesmas, no sistema de agendamento, no monitoramento, na atenção programada e na continuidade do cuidado (MOYSÉS; SILVEIRA FILHO; MOYSÉS, 2012). Melhorias realizadas têm sido associadas à redução dos fatores de risco para doença cardiovascular e diabetes (PARCHMAN; KAISSI, 2009).

A segunda equipe, apesar de todos os seus profissionais estarem presentes durante a aplicação do instrumento, demonstrou-se pouco participativa, sob a influência de uma liderança na qual se faziam prevalecer as notas atribuídas aos componentes do instrumento ACIC (tabela 1).

$\mathrm{O}$ estilo de liderança apresentado nesta equipe é conhecido como autoritário, no qual o líder posiciona-se no topo, com uma conduta em que há ausência de incertezas, sendo sua posição considerada como a única verdade. Esse comportamento pode ter contribuído para que o resultado da equipe tenha sido a maior média dentre as demais 
(tabela 1) (LANZONI; MEIRELLES, 2011).

Esta equipe considerou, como maior nota, a segunda dimensão - articulação com a comunidade -, por ponderar como melhores componentes a articulação das instituições de saúde e das pessoas usuárias com os recursos comunitários, as parcerias com as organizações comunitárias e o trabalho articulado do agente comunitário de saúde (quadro 1).

Apesar de as equipes avaliadas referirem alguma articulação com a comunidade (tabela 2), os vínculos comunitários são práticas raras, o que justifica alguns estudos não incluírem informações detalhadas sobre esta dimensão em suas pesquisas, ao utilizarem o ACIC (STRICKLAND ET AL., 2010). Demandas dos cuidados agudos muitas vezes impediram o desenvolvimento de relações comunitárias, contudo, esses vínculos foram especificamente relacionados a um melhor desempenho no processo de atendimento e, consequentemente, a melhores resultados (SI ET AL., 2005; PARCHMAN; KAISSI, 2009).

Quanto à terceira equipe, o instrumento foi aplicado para quatro membros, que se apresentaram participativos, demonstrando respeito às opiniões individuais e conhecimento em relação às condições crônicas (tabela 1). A liderança do enfermeiro pode ter influenciado os demais a atribuírem à primeira dimensão - organização da atenção à saúde - a maior nota (tabela 2).

Estudos demonstram que a melhoria dos resultados na pontuação ACIC para a primeira dimensão exige uma grande mudança na organização dos cuidados de saúde, o que depende da gestão da equipe, porém são necessárias mais pesquisas que expliquem a associação entre a avaliação ACIC e a mudança organizacional (SUNAERT ET AL., 2009).

A quarta equipe diferenciou-se das demais, tanto em relação ao tempo de aplicação do instrumento (20 minutos) quanto com sua insatisfação em relação à liderança da UBSF.

No que diz respeito ao tempo empregado para a aplicação do instrumento, constatou-se que a média de resposta foi de aproximadamente 1 hora e 30 minutos, com exceção da equipe 4. Pesquisas apontam um resultado que varia de 15 a 30 minutos, podendo chegar a 2 horas (BONOMI ET AL., 2002; MOYSÉS; SILVEIRA FILHO; MOYSÉS, 2012; SI ET AL., 2005; SUNAERT ET AL., 2009).

Nesta equipe, o instrumento foi aplicado junto aos três profissionais mínimos exigidos nos critérios de inclusão da pesquisa, que se apresentaram com uma postura negativa e desinteressada. A insatisfação dos profissionais pode estar associada à pouca experiência da gestão, refletida durante a aplicação do instrumento.

Todos estes fatores podem ter contribuído para o baixo nível de pontuação desta equipe em relação às demais, resultando uma capacidade básica para atenção às condições crônicas.

No que se refere à aplicabilidade do instrumento, estudos demonstram que o número ideal de participantes deve ser, no mínimo, três (BONOMI ET AL., 2002). Neste estudo, observou-se que, nas equipes que participaram com maior número de profissionais - equipes 1,2 e 3 -, as notas tenderam a ser maiores (tabelas 1 e 2). Porém, estudos apontam que, após a aplicação ter sido feita individualmente com três participantes, optou-se, em um segundo momento, por envolver um maior número de profissionais e estabelecer um consenso (MOYSÉS; SILVEIRA FILHO; MOYSÉS, 2012; SUNAERT ET AL., 2009).

Para esta equipe, a quinta dimensão desenho do sistema de prestação de serviços - apresentou a maior nota, sendo a liderança da equipe, o monitoramento e a atenção programada à condição crônica os componentes com maior pontuação (tabela 2).

A quinta equipe também participou, somente com os três profissionais exigidos (tabela 1), sendo que a liderança apresentou-se atuante, com comportamento semelhante ao encontrado nas equipes 1 e 3 . Ainda sobre liderança, constatou-se, nas equipes 1 , 
3 e 5, aquela conhecida como autêntica, que mantém todos os membros unidos, oportuniza uma visão compartilhada dos objetivos e envolve os profissionais nas decisões da equipe (LANZONI; MEIRELLES, 2011).

Esta equipe pontuou a terceira dimensão - autocuidado - como a melhor nota (tabela 2). Esta dimensão trata da forma como a equipe pode desenvolver a capacidade das pessoas e familiares para lidar com os desafios de conviver e tratar a condição crônica, além de reduzir as complicações e os sintomas da doença. Pacientes que desenvolvem o comportamento para o autocuidado têm fatores de risco mais bem controlados (PARCHMAN; KAISSI, 2009).

$\mathrm{O}$ autocuidado apoiado também foi referenciado com boas notas pelas equipes 2,3 e 5 (tabela 2), as quais demonstraram maior conhecimento em relação às condições crônicas, no que se refere ao acolhimento das preocupações das pessoas usuárias e seus familiares, e às intervenções efetivas, que levam a mudanças de comportamento dos usuários e de suporte de pares (quadro 1) (MENDES, 2012; MOYSÉS; SILVEIRA FILHO; MOYSÉS, 2012).

No entanto, em relação à quinta equipe, destaca-se a baixa nota que apresentou para a sétima dimensão - integração dos componentes do MACC -, diferenciando-se das demais (tabela 2). Esta dimensão traz aspectos que devem combinar com todos os elementos do modelo, como, por exemplo, associar as metas de autocuidado com os registros no sistema de informação, ou associar as políticas locais com as atividades dos planos de cuidado dos usuários (locais para o desenvolvimento de atividades físicas, estruturação de hortas comunitárias etc.) (MOYSÉS; SILVEIRA FILHO; MOYSÉS, 2012).

Esta baixa pontuação pode estar relacionada à reduzida compreensão em relação aos conceitos de diretrizes clínicas, plano de cuidado e a associação entre os elementos do modelo de atenção.

A experiência da Secretaria Municipal de Saúde de Curitiba (PR) demonstrou que, durante a aplicação do instrumento, alguns profissionais também manifestaram dificuldade em relação a determinados conceitos-chave para o desenvolvimento de atenção às condições crônicas, como, por exemplo: diretrizes clínicas, autocuidado, plano de cuidado (MOYSÉS; SILVEIRA FILHO; MOYSÉS, 2012).

Esta mesma situação foi observada durante a aplicação do instrumento nesta pesquisa. O ACIC tem o potencial de ser afetado pelo papel de uma pessoa, do trabalho, do local e da compreensão dos elementos do MCC (SOlberg ET AL., 2006). Itens como a organização do sistema de saúde, o autocuidado e o desenho de prestação de serviço foram os de mais difícil entendimento (STEINHAEUSER ET AL., 2011).

Por esta razão, recomenda-se que, antecedendo sua aplicação, seja realizado um pré-teste junto aos profissionais locais, para verificar a necessidade de revisão e adaptação na escrita dos seus componentes, de modo a facilitar a compreensão e a discussão do mesmo.

Com relação às dimensões, o sistema de informação clínica - sexta dimensão - apresentou notas baixas para todas as equipes (tabela 2). Esta dimensão demonstra que uma informação útil e personalizada, por cada usuário e pelas populações usuárias com condições crônicas é um aspecto crítico de modelos de atenção efetivos, especialmente daqueles que empregam abordagens populacionais (MOYSÉS; SILVEIRA FILHO; MOYSÉS, 2012).

O Hygia, sistema de informação disponibilizado pela Secretaria Municipal de Saúde de Campo Grande (MS), integra todos os pontos de atenção da rede municipal de saúde, no entanto, não contempla os elementos necessários para a gestão do cuidado das condições crônicas: não é integrado à cogestão do paciente em termos de documentar as metas e atividades, e apresenta pouca articulação entre a informação e os serviços comunitários e clínicos. Outros estudos demonstram a mesma situação (PARCHMAN; KAISSI, 
2009; SI ET AL., 2005; STEURER-STEY ET AL., 2012).

A utilização de sistemas de informação que incluam o uso de lembretes, alertas, relatórios e feedbacks do desempenho clínico possibilita um atendimento clínico estruturado e melhoria na qualidade dos cuidados, pois o sistema de informação tem três funções importantes: (1) registro de uma população-alvo; (2) fornecimento de lembretes para as equipes de APS, a fim de cumprir as diretrizes do cuidado; e (3) estabelecimento de informações relevantes para a qualidade do atendimento, sendo este o menos desenvolvido (STRICKLAND ET AL., 2010; SI ET AL., 2005; STEURER-STEY ET AL., 2012). O aumento na pontuação do ACIC nesta dimensão foi associado a uma considerável melhoria na prestação de serviços (PARCHMAN; KAISSI, 2009).

O total de todas as dimensões demonstra uma razoável capacidade de atenção às condições crônicas para as equipes avaliadas neste estudo (tabela 2). Conforme a orientação do próprio instrumento, é comum que algumas equipes deem início a um processo de mudança com média menor do que ' 5 ' em algumas ou em todas as dimensões do instrumento. Também é comum as equipes acreditarem que proporcionam mais atenção às condições crônicas do que realmente acontece (MOYSÉS; SILVEIRA FILHO; MOYSÉS, 2012).

Estudos demonstram que o mesmo ocorre com a reaplicação do instrumento: depois de transcorrido determinado período para comparação com a linha de base, algumas variáveis podem apresentar discreto aumento; ou, ao contrário, ao reavaliar sua capacidade institucional, a equipe pode atribuir uma redução no desempenho por demonstrar postura autocrítica (SCHWAB ET AL., 2014).

Há dificuldades, em curto prazo, de melhorias em todas as dimensões do MCC, porém, para alinhar e conseguir as mudanças necessárias, é preciso colaboração e incentivo de todos os atores (STEURER-STEY ET AL.,
2012).

\section{Considerações finais}

O ACIC se apresentou como uma importante ferramenta, que possibilitou avaliar a percepção dos profissionais quanto à capacidade institucional para atenção às condições crônicas.

No que se refere à sua aplicação, mesmo este estudo tendo obedecido à mesma metodologia de aplicação de estudos anteriores, que sugerem a participação de, no mínimo, três participantes, verificou-se a necessidade de ser realizado com todos os profissionais da equipe. Por isso, recomenda-se aplicá-lo inicialmente de maneira individualizada e após fomentar a discussão em equipe, para posterior consenso, a fim de se garantir que as percepções de todos os participantes possam ser discutidas.

A média das equipes resultou como uma capacidade razoável para atenção às condições crônicas, sendo o sistema de informação clínica a principal fragilidade e o desenho do sistema de prestação de serviços de saúde, a de maior potencial.

A utilização do ACIC em todas as equipes de APS deve ser estimulada, para o planejamento de projetos e ações que propiciem a melhoria da qualidade da atenção às condições crônicas, sendo esta a principal vantagem da aplicabilidade deste instrumento. Sugere-se que sejam realizados estudos que analisem a percepção dos usuários, por meio do instrumento Avaliação do Usuário sobre o Cuidado às Condições Crônicas (Patient Assessment of Chronic Illness Care - PACIC), que poderá proporcionar uma avaliação mais completa, uma vez que a corresponsabilização do usuário influencia a atenção ofertada, principalmente no tocante ao autocuidado, um dos componentes do MACC, já que o ACIC limita-se à percepção do profissional de saúde. 


\section{Referências}

ALWAN, A. et al. Monitoring and surveillance of chronic noncommunicable diseases: progress and capacity in high-burden countries. Lancet, London, v. 376, n. 9755, p. 1861-1868, 2010. Disponível em: <http://www.ncbi.nlm. nih.gov/pubmed/21074258>. Acesso em: 10 ago. 2014.

BONOMI, A. E. et al. Assessment of chronic illness care (ACIC): a pratical tool to measure quality improvement. Health Services Research, Chicago, v. 37, n. 3, p. 791-820, 2002. Disponível em: <http://www.ncbi.nlm.nih.gov/ pmc/articles/PMC1434662/>. Acesso em: 10 ago. 2014.

BOWEN, J. L. et al. Assessing chronic illness care education (ACIC-E): a tool for tracking education redesign for improving chronic care education. Journal of General Internal Medicine, Philadelphia, v. 25, supl. 4, p. 593-609, 2010. Disponível em: <http://www.ncbi.nlm. nih.gov/pmc/articles/PMC2940447/>. Acesso em: 10 ago. 2014.

BRASIL. Ministério da Saúde. Conselho Nacional de Secretários de Saúde. Planificação da atenção primária à saúde nos estados. Brasília, DF: Conselho Nacional de Secretários de Saúde, 2011.

Ministério da Saúde. Gabinete do Ministro. Portaria GM n ${ }^{\circ} 483$, de $1^{\circ}$ de abril de 2014. Redefine a Rede de Atenção à Saúde das Pessoas com Doenças Crônicas no âmbito do Sistema Único de Saúde (SUS) e estabelece diretrizes para a organização das suas linhas de cuidado. Diário Oficial [da] União, Brasília, DF, seção 1, 2 abr. 2014. Disponível em: <http://bvsms.saude.gov. br/bvs/saudelegis/gm/2014/prt0483_01_04_2014.html>. Acesso em: 8 set. 2014 .

Ministério da Saúde. Portaria GM no 4.279, de 30 de dezembro de 2010. Estabelece diretrizes para a organização da rede de atenção à saúde no âmbito do sistema único de saúde (SUS). Diário Oficial [da] União, Brasília, DF, 31 dez. 2010. Disponível em: <http:// portal.saude.gov.br/oirtal/arquivos/pdf/portaria4279_ docredes.pdf>. Acesso em: 05 jun. 2014.

Ministério da Saúde. Secretaria de Atenção à Saúde. Departamento de Atenção Básica. Política Nacional de Atenção Básica. Brasília, DF: Ministério da Saúde, 2012.

CRAMM, J. M. et al. Development and validation of a short version of the assesment of chronic illness care (ACIC) in dutch disease management programs. Health and Quality of Life Outcomes. London, v. 9, n. 49, p. 49-58, 2011. Disponível em: <http://www.hqlo.com/ content/9/1/49>. Acesso em: 10 ago. 2014.

DAHLGREN, G.; WHITEHEAD, M.; Policies and strategies to promote social equity in health. Background document to WHO - Strategy paper for Europe. Arbetsrapport/Institutet for Framtidsstudier, Stockolm, v. 2007, n. 14, 1991. Disponível em: <http://econpapers. repec.org/paper/hhsifswps/2007_5fo14.htm>. Acesso em: 10 ago. 2014

DUARTE, E. C.; BARRETO, S. M. Transição demográfica e epidemiológica: a epidemiologia e serviços de saúde revisita e atualiza o tema. Epidemiol. Serv. Saúde, Brasília, DF, v. 21, n. 4, p. 529-532, dez. 2012. Disponível em: <http://scielo.iec.pa.gov.br/scielo.php?script=sci arttext\&pid=S1679-49742012000400001\&lng=pt\&nrm=i so>. Acesso em: 2 fev. 2014.

GOMUTBUTRA, P. et al. The reliability and validity of thai version of assessment of chronic illness care (ACIC). Journal of the Medical Association of Thailand, Bangkok, v. 95, n. 8, p. 1105-1113, ago. 2012. Disponível em: <http:// www.ncbi.nlm.nih.gov/pubmed/23061317>. Acesso em: 10 ago. 2014.

LANZONI, G. M. M.; MEIRELLES, B. H. S. Liderança do enfermeiro: uma revisão integrativa da literatura. Rev. Latino-Am. Enfermagem, Ribeirão Preto, v. 19, n. 3, p. 651-658, jun. 2011. Disponível em: <http://www.scielo.br/ scielo.php?pid=S0104-11692011000300026\&script=sci arttext\&tlng=pt.http://dx.doi.org/10.1590/S0104$11692011000300026>$. Acesso em: 10 ago. 2014.

MACINKO, J.; DOURADO, I.; GUANAIS, F. C. Doenças crônicas, atenção primária e desempenho dos sistemas de saúde: diagnósticos, instrumentos e intervenções. Banco Interamericano de Desenvolvimento (BID), 2011.

MENDES, E. V. O cuidado das condições crônicas na atenção primária à saúde: o imperativo da consolidação da estratégia da saúde da família. Brasília, DF: Organização Pan-Americana da Saúde, 2012.

MOYSÉS, S. T.; SILVEIRA FILHO, A. D.; MOYSÉS, S. (Org.). Laboratório de inovações no cuidado das condições crônicas na APS: a implantação do modelo de atenção às condições crônicas na UBS Alvorada em Curitiba, Paraná. Brasília, DF: Organização Pan-Americana da Saúde, 2012.

PARCHMAN, M.; KAISSI, A. A. Are elements of the chronic care model associated with cardiovascular risk factor control in type 2 diabetes? Jt Comm J Qual Patient 
Saf, Oak Brook, v. 35, n. 3, p. 133-138, 2009. Disponível em: <http://www.ncbi.nlm.nih.gov/pubmed/19326804>. Acesso em: 10 ago. 2014.

SCHWAB, G. L. et al. Percepção de inovações na atenção às doenças/condições crônicas: uma pesquisa avaliativa em Curitiba. Saúde Debate, Rio de Janeiro, v. 38, n. esp., p. 307-318, out. 2014. Disponível em: <http://www.scielo. br/pdf/sdeb/v38nspe/0103-1104-sdeb-38-spe-0307.pdf $>$. Acesso em: 12 mar. 2015.

SI, D. et al. Assessment health centre systems for guiding improvement in diabetes care. BMC Health Services Research, London, v. 5, n. 56, p. 1- 23, 2005. Disponível em: <http://www.ncbi.nlm.nih.gov/pmc/articles/ PMC1208882/>. Acesso em: 10 ago. 2014.

SOLBERG, L. I. et al. Care quality and implementation of the chronic care model: a quantitative study. Annals of Family Medicine, Leawood, v. 4, n. 4, p. 310-316, 2006. Disponível em: <http://www.ncbi.nlm.nih.gov/ pubmed/16868234>. Acesso em: 10 ago. 2014.

STEINHAEUSER, J. et al. Applicability of the assessment of chronic illness care (ACIC) instrument in germany resulting in a new questionnaire: questionnaire of chronic illness care in primary care. BMC Health Services Research, London, v. 11, n. 164, jul. 2011. Disponível em: $<$ http://www.biomedcentral.com/14726963/11/164>. Acesso em: 10 ago. 2014.

STEURER-STEY, C. et al. The german version of the assessment of chronic illness care: instrument translation and cultural adaptation. Eval Clin Pract, UK, v. 18, n. 1, p. 1-4, 2012. Disponível em: <http://www.ncbi.nlm.nih.gov/ pubmed/20698920>. Acesso em: 10 ago. 2014.

STRICKLAND, P. A. O. et al. Features of the Chronic Care Model associated with behavioral counseling and diabetes care in community primary care. J Am Board Fam Med, Cuba, v. 23, n. 3, p. 295-305, 2010. Disponível em: <http://www.ncbi.nlm.nih.gov/pmc/articles/ PMC2924566/>. Acesso em: 10 ago. 2014.

SUNAERT, P. et al. Implementation of a program for type 2 diabetes based on the Chronic Care Model in a hospital-centered health care system: 'the Belgian experience'. BMC Health Services Research, London, v. 9, n. 152, p. 1-14, ago. 2009. Disponível em: <http://www. ncbi.nlm.nih.gov/pmc/articles/PMC2757022/>. Acesso em: 10 ago. 2014.

WAGNER, E. H. Chronic disease management: what will it take to improve care for chronic illness? Effective Clinical Practice, Philadelphia, v. 1, n. 1, p. 2-4, ago./set. 1998. Disponível em: <http://www.ncbi.nlm.nih.gov/ pubmed/10345255>. Acesso em: 10 ago. 2014.

Recebido para publicação em julho de 2015

Versão final em novembro de 2015

Conflito de interesses: inexistente

Suporte financeiro: não houve 